\title{
Incidence and risk factor analysis for sarcopenia in patients with cancer
}

\author{
GUOXING ZHANG $^{1^{*}}$, XIUJIANG LI ${ }^{1 *}, \mathrm{CHANGPING} \mathrm{SUI}^{2}, \mathrm{HUI}^{*} \mathrm{ZHAO}^{1}$, \\ JIHONG ZHAO ${ }^{2}$, YUE HOU ${ }^{3}$ and YUJUN DU ${ }^{3}$ \\ ${ }^{1}$ Intensive Care Unit; ${ }^{2}$ Department of Radiology, Jilin Province Tumor Hospital, Changchun, Jilin 130001; \\ ${ }^{3}$ Department of Urology, Center of Nephrology, The First Hospital of Jilin University, Changchun, Jilin 130021, P.R. China
}

Received January 28, 2015; Accepted October 9, 2015

DOI: $10.3892 / \mathrm{ol} .2015 .4019$

\begin{abstract}
The objective of the present study was to investigate the incidence of and possible risk factors associated with sarcopenia among cancer patients. Patients with cancer were examined through the use of lumbar magnetic resonance imaging, and clinical data was collected between September and December, 2012, at Jilin Province Tumor Hospital (Changchun, China). The data was subsequently compared between patients with and without sarcopenia. Of the 113 treated cancer patients, 96 patients [39 males (L3 index, $<52.4 \mathrm{~cm}^{2} / \mathrm{m}^{2}$ ) and 57 females (L3 index, $<38.5 \mathrm{~cm}^{2} / \mathrm{m}^{2}$ )] suffered from sarcopenia. Overall, the development of sarcopenia was not significantly associated with patient age or treatment, including surgery, chemotherapy or radiotherapy $(\mathrm{P}>0.05)$. The frequency of treatment-associated complications did not differ significantly between patients with or without sarcopenia. However, males were more inclined to develop sarcopenia than females $(\mathrm{P}=0.02)$. Patients with sarcopenia had significantly less lymphocytes than patients without sarcopenia $(\mathrm{P}=0.03)$. This was confirmed through multiple logistic regression analyses $(\mathrm{P}=0.046)$, which also identified that patients with cancer with an Eastern Cooperative Oncology Group score $>2$ had a significantly increased risk of developing sarcopenia. Finally, the serum albumin level in sarcopenia patients was $36.18 \pm 4.65 \mathrm{~g} / \mathrm{l}$, which was not significantly less than that of patients without sarcopenia (39.67 $\pm 3.69 \mathrm{~g} / \mathrm{l} ; \mathrm{P}=0.11)$. The incidence of sarcopenia among
\end{abstract}

Correspondence to: Dr Yujun Du, Department of Urology, Center of Nephrology, The First Hospital of Jilin University, 71 Xinmin Street, Changchun, Jilin 130021, P.R. China

E-mail:kjkdyj@163.com

*Contributed equally

Abbreviations: ECOG, Eastern Cooperative Oncology Group; $\mathrm{OR}$, odds ratio; $\mathrm{CI}$, confidence interval

Key words: lymphocytes, cluster of differentiation 4, cancer, sarcopenia, cluster of differentiation 8 patients with cancer is high, particularly for males. Further research with larger sample sizes would be beneficial, with the aim of verifying the results obtained in the present study. During the treatment of patients with sarcopenia, precaution should continue to be taken to prevent associated complications, including infection, diarrhea and myelosuppression.

\section{Introduction}

Sarcopenia is defined by symptoms of decreased muscle strength, fatigue and metabolic disorders initiated by a reduction in skeletal muscle mass, which is characterized by muscle atrophy and the reduction of muscle tissue quality. Muscle fibers are replaced by fibrotic tissue, resulting in increased frailty and deterioration of muscle function, with neuromuscular junction degeneration and alterations in oxidative stress and muscle metabolism observed (1). Currently, there is no generally accepted definition of sarcopenia due to a lack of clinical awareness of the signs and consequences (2). A clinical definition and diagnostic criteria, regarding age-associated sarcopenia, was established by the European Working Group on Sarcopenia in Older People, focusing on the presence of reduced muscle mass and function, including performance and strength (3).

As the degenerative loss of skeletal muscle mass, quality and strength, sarcopenia is primarily associated with aging. It is reported that $0.5-1.0 \%$ of skeletal muscle mass is lost per year beyond the age of 25 years (4). Furthermore, the incidence of sarcopenia is associated with the diagnosis of cancer. Cancer is possibly the most notable pathological condition that promotes muscle atrophy, particularly in elderly patients (5). The incidence of cancer and subsequent survivorship is observed to be greatest in patients $>60$ years of age $(6,7)$. The probability of developing melanoma, non-Hodgkin lymphoma, or lung, prostate, colon, uterine, leukemia or breast cancer increases with age (8). Therefore, the older the sarcopenia patient, the more likely they are to encounter a considerable reduction in body mass preceding and following a diagnosis of cancer. In comparison to healthy, age-matched individuals, a similar pattern of muscle deterioration in older adults may advance the progression of sarcopenia at an increased rate. Cachexia, a severe condition during which the body mass of an individual greatly decreases, with a proportional reduction also observed 
in adipose and muscle tissue, is frequently associated with cancer-associated muscle atrophy. Additionally, cachexia-associated cytokines are able to cross the blood brain barrier and modify the activity of hunger regulatory systems (9). As a result, cancer patients with cachexia often develop anorexia, with the incidence rate ranging from $15-40 \%$ (10). Anorexia is a major contributor to muscle wasting and is believed to originate from increased levels of, and sensitivity to, cytokines.

With respect to the potential mechanisms of sarcopenia in patients with cancer, the most important is the use of endogenous amino acids by the liver to produce antitumor proteins through the consumption of skeletal muscle in an inefficient manner, with $2.6 \mathrm{~g}$ of muscle protein required to synthesize $1 \mathrm{~g}$ of fibrinogen (11). Furthermore, pro-inflammatory cytokines trigger cancer cachexia (12), which appears to specifically target skeletal muscle given that the visceral protein compartment is preserved even once patients have lost $30 \%$ of their body weight (13). Bryant et al (14) indicated that tumor necrosis factor- $\alpha$ (TNF- $\alpha$ ) is highly specific when identifying myosin heavy chains for breakdown in tumor models of wasting and in cell culture. Moreover, food intake is suppressed when TNF- $\alpha$ and interleukin-1 are administered either centrally or peripherally (15). Alongside a decrease in caloric intake, an increase in resting energy expenditure (REE) may also contribute to cancer-associated muscle atrophy. A high prevalence of hypermetabolism is demonstrated among patients with lung and pancreatic cancer, suggesting it may be cancer-type specific (16). By contrast, considerable changes in metabolism are not commonly identified in patients with colorectal or gastric cancer (16).

Cancer patients with sarcopenia typically have a poor prognosis with regard to complications commonly associated with cancer treatment. Furthermore, the tolerance to surgery, chemotherapy and radiotherapy is decreased in patients with cancer and sarcopenia. To investigate the incidence of sarcopenia and possible risk factors in patients with cancer, the present study reviewed and compared the clinical data of patients with cancer for whom lumbar magnetic resonance imaging (MRI) analysis was performed at Jilin Province Tumor Hospital (Changchun, China). A high incidence of sarcopenia was observed in the patients with cancer, particularly among those with later stages of cancer and those treated with chemotherapy. A non-significantly higher rate of severe complications among cancer patients with sarcopenia was observed. The data indicate that prevention of treatment-associated complications, including infection, diarrhea and myelosuppression, is important in cancer patients with sarcopenia.

\section{Materials and methods}

Patients. All patients who were diagnosed with any type of cancer and received treatment in the form of surgery, chemotherapy or radiotherapy at Jilin Province Tumor Hospital were screened. Following the exclusion of patients who did not receive any treatment, a total of 113 patients treated between September and December, 2012, were included in the present study. Among these, 41 patients were male, whilst 72 patients were female. The average age of the patients was $58.43 \pm 11.04$ years (range, 23-84 years). The study protocol
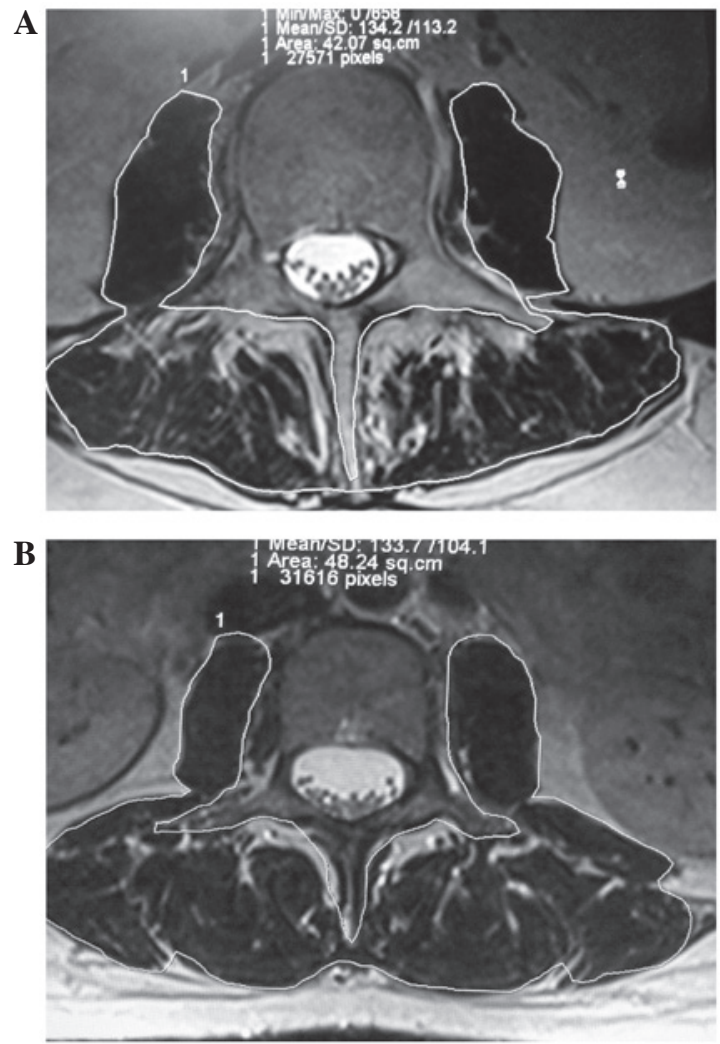

Figure 1. Representative magnetic resonance images used for measurement of the L3-sectional muscle area in cancer patients (A) with sarcopenia and (B) without sarcopenia

was reviewed and approved by the Institutional Review Board of Jilin Province Tumor Hospital, and written informed consent was obtained from all participants.

Data collection. Data regarding patient age, gender, cancer type, tumor stage, Eastern Cooperative Oncology Group (ECOG) score, albumin level in serum, cluster of differentiation (CD)4 and CD8 levels, lymphocyte counts, therapeutic techniques applied (including surgery, chemotherapy and radiotherapy) and any complications experienced during treatment (including infection, diarrhea and myelosuppression) were obtained from the patients' medical records.

Two directly measured body composition variables were assessed in the current study: Total lumbar skeletal muscle cross-sectional area $\left(\mathrm{CSA} ; \mathrm{cm}^{2}\right)$ and total lumbar adipose tissue area $\left(\mathrm{cm}^{2}\right)$. These body composition variables are indicated to be linearly associated with the whole-body muscle and adipose tissue masses, respectively $(17,18)$. Given that muscle and adipose tissue are clearly exhibited on MRI, the areas of muscle and adipose tissue in lumbar cross-sections are linearly correlated with those of the whole body (19). Thus, measurements of lumbar muscle and adipose tissue areas within cross-sectional MR images may be used to estimate the levels of body muscle and fat. Antoun et al (20) demonstrated that a sarcopenia diagnosis may be made based on measurements of the L3-sectional muscle area divided by the patient's body surface area.

The L3-sectional muscle area was measured for all patients through the use of MRI (MAGNETOM Espree 1.5T; 
Table I. Clinical characteristics of patients with and without sarcopenia.

\begin{tabular}{|c|c|c|c|}
\hline Variable & Sarcopenia & Non-sarcopenia & P-value \\
\hline Mean age $\pm \mathrm{SD}$, years & $57.82 \pm 12.07$ & $58.24 \pm 11.77$ & 0.81 \\
\hline Gender, n (\%) & & & 0.02 \\
\hline Male & $39(40.63)$ & $2(11.76)$ & \\
\hline Female & $57(59.38)$ & $15(88.24)$ & \\
\hline Body mass index, $\mathrm{kg} / \mathrm{m}^{2}$ & $23.16 \pm 3.53$ & $23.46 \pm 4.21$ & 0.20 \\
\hline Cancer stage, n (\%) & & & 0.36 \\
\hline Early stage (I-III) & $34(35.42)$ & $8(47.06)$ & \\
\hline Advanced stage (IV) & $62(64.58)$ & $9(52.94)$ & \\
\hline Surgical procedure, n (\%) & & & 0.30 \\
\hline No & $47(48.96)$ & $6(26.42)$ & \\
\hline Yes & $49(51.04)$ & $11(35.29)$ & \\
\hline Chemotherapy, n (\%) & & & 0.72 \\
\hline No & $19(19.79)$ & $4(23.53)$ & \\
\hline Yes & $77(80.21)$ & $13(76.47)$ & \\
\hline Radiotherapy, n (\%) & & & 0.76 \\
\hline No & $47(48.96)$ & $9(52.94)$ & \\
\hline Yes & $49(51.04)$ & $8(47.06)$ & \\
\hline \multicolumn{4}{|l|}{ Blood analysis, mean $\pm \mathrm{SD}$} \\
\hline Albumin, g/l & $36.71 \pm 4.65$ & $39.67 \pm 3.69$ & 0.11 \\
\hline $\mathrm{CD} 4, \%$ & $30.47 \pm 4.74$ & $32.71 \pm 5.75$ & 0.92 \\
\hline $\mathrm{CD} 8, \%$ & $22.97 \pm 4.74$ & $23.00 \pm 2.74$ & 0.07 \\
\hline $\mathrm{CD} 4 / \mathrm{CD} 8, \%$ & $1.44 \pm 0.29$ & $1.38 \pm 0.37$ & 0.54 \\
\hline Lymphocytes, $\sim 10^{9} / 1$ & $1.12 \pm 0.51$ & $1.27 \pm 0.74$ & 0.03 \\
\hline Complications, n (\%) & & & 0.57 \\
\hline No & $73(76.04)$ & $14(82.35)$ & \\
\hline Yes & $23(23.96)$ & $3(17.65)$ & \\
\hline Eastern Cooperative Oncology Group score, n (\%) & & & 0.19 \\
\hline$\leq 1$ & $78(81.25)$ & $16(94.12)$ & \\
\hline$>2$ & $18(18.75)$ & $1(5.88)$ & \\
\hline
\end{tabular}

$\mathrm{SD}$, standard deviation; $\mathrm{CD}$, cluster of differentiation.

Siemens AG, Munich, Germany). Representative MR images employed for the measurement of the L3-sectional muscle area in the cancer patients with or without sarcopenia are presented in Fig. 1. Prado et al (21) determined gender-specific cut-offs for sarcopenia significantly associated with mortality in patients with solid tumors, and these cut-offs of $52.4 \mathrm{~cm}^{2} / \mathrm{m}^{2}$ for males and $38.5 \mathrm{~cm}^{2} / \mathrm{m}^{2}$ for females were utilized in the present study. Patients with muscle areas below these cut-off values were classified as having sarcopenia.

Statistical analysis. Statistical analysis was performed using SPSS software, version 19.0 (IBM SPSS, Armonk, NY, USA). Normally distributed data are presented as the mean \pm standard deviation, and comparisons between two means were conducted using Student's t-test for unpaired data. The prevalence of sarcopenia is presented as a frequency and was compared between groups using the $\chi^{2}$ test. Relative risks were estimated as an odds ratio with a $95 \%$ confidence interval (CI). Risk factors associated with sarcopenia in cancer patients were investigated via multiple logistic regression analyses using the forward step-wise method. Two-tailed $\mathrm{P}<0.05$ was considered to indicate a statistically significant difference.

\section{Results}

Of the 113 patients identified, 96 were classified as having sarcopenia [39 males (L3 index, $<52.4 \mathrm{~cm}^{2} / \mathrm{m}^{2}$ ) and 57 females ( $\mathrm{L} 3$ index, $<38.5 \mathrm{~cm}^{2} / \mathrm{m}^{2}$ )]. The results indicated that males were more likely to develop sarcopenia (Table I). The average age of patients who developed sarcopenia was $57.82 \pm 12.07$ years, which did not differ significantly from that of patients without sarcopenia $(58.24 \pm 11.77$ years) $(\mathrm{P}=0.81)$ (Table I). The incidence of sarcopenia among stage IV cancer patients was $87.3 \%(62 / 71)$, which was slightly higher than that observed in stage I-III patients $(81.0 \% ; 34 / 42)$. However, this difference was not statistically significant (Table I).

Among the patients in the current study, no significant associations were identified between the development of 
Table II. Results of multivariate logistic regression analyses for the effect of clinical variables on sarcopenia development among 113 patients with cancer.

\begin{tabular}{|c|c|c|c|}
\hline Variable & Adjusted OR ${ }^{\mathrm{a}}$ & $95 \% \mathrm{CI}$ & P-value \\
\hline \multicolumn{4}{|l|}{ ECOG score } \\
\hline$\leq 1$ & 1.00 & & \\
\hline$>2$ & 11.60 & $3.01-44.75$ & $<0.001$ \\
\hline \multicolumn{4}{|l|}{ Lymphocytes, $\sim 10^{9} / 1$} \\
\hline Normal (0.8-4.0) & 1.00 & & \\
\hline Abnormal $(<0.4)$ & 8.31 & $0.99-69.44$ & 0.046 \\
\hline
\end{tabular}

sarcopenia and treatment, including surgery, chemotherapy or radiotherapy $(\mathrm{P}>0.05)$. The incidence of sarcopenia was $81.7 \%$ (49/60) among patients who received surgical treatment and $88.7 \%$ (47/53) among patients who did not undergo surgical treatment. With regard to chemotherapy, the incidence of sarcopenia among patients was slightly, but insignificantly, higher than that of patients not treated with chemotherapy $[85.6 \%$ (77/90) vs. $82.6 \%(19 / 23) ; \mathrm{P}=0.72]$. Additionally, the development of sarcopenia was also not associated with treatment by radiotherapy $(\mathrm{P}=0.76)$, with $86.0 \%(49 / 57)$ of patients who received radiotherapy and $83.9 \%(47 / 56)$ of patients who did not receive radiotherapy developing sarcopenia. The frequency of complications, including infection, diarrhea and myelosuppression, during treatment was not significantly greater among the patients with sarcopenia $(24.0 \% ; 23 / 96)$ compared with patients without sarcopenia $(17.7 \% ; 3 / 17)(\mathrm{P}=0.57)$ (Table I).

Albumin levels in serum may reflect the nutritional status of patients. In the present study, a non-significant association was observed between the serum albumin level and the development of sarcopenia $(\mathrm{P}=0.11)$. The mean serum albumin level in the patients with sarcopenia was $36.18 \pm 4.65 \mathrm{~g} / \mathrm{l}$, which was lower than that in patients without sarcopenia $(39.67 \pm 3.69 \mathrm{~g} / \mathrm{l})$. Additionally, a non-significant association between CD8 expression and the development of sarcopenia was also identified $(\mathrm{P}=0.07)$ (Table I). Notably, a statistically significant association between the number of lymphocytes and the development of sarcopenia was observed $(\mathrm{P}=0.03)$.

Multiple logistic regression analyses demonstrated that the patients with sarcopenia had significantly fewer lymphocytes than the patients without sarcopenia $(\mathrm{P}=0.046)$, and patients with cancer with an ECOG score $>2$ had a significantly increased risk of developing sarcopenia (Table II). However, further research with larger sample sizes is required to verify these findings, due to the wide ranges of the confidence intervals (ECOG score: 95\% CI, 3.01-44.75; lymphocyte count: 95\% CI, 0.99-69.44).

\section{Discussion}

In the present study, the development of sarcopenia was not significantly associated with treatment, including surgery, chemotherapy or radiotherapy. However, it was observed that male patients with cancer were more likely to develop sarcopenia, and patients with sarcopenia also had significantly lower lymphocyte counts than patients without sarcopenia.

Skeletal muscle is the body's protein pool, with $60 \%$ of proteins stored within it in various forms. Skeletal muscle protein is widely mobilized into amino acids to provide to the body when under stress. Sarcopenia may be caused by excessive muscle consumption associated with aging, cancer and malnutrition. Increasing evidence indicates that a number of factors may lead to the development of sarcopenia, including cachexia and malnutrition, and sarcopenia has been associated with metastasis and a poor prognosis in patients with cancer (22). Therefore, it is necessary to investigate the relative risk factors for sarcopenia in order to improve the overall survival and quality of life of individuals who develop this condition.

In the general population, age is a leading factor for the progression of sarcopenia. Through three-dimensional imaging techniques, including CT, MRI and other measures, the muscle CSA has been observed to decrease by $\sim 40 \%$ from 20 to 60 years old (23). In the present study, the average age of patients with sarcopenia was higher than that of patients without sarcopenia, but this difference was not statistically significant, indicating that age was not a significant risk factor for sarcopenia among patients with cancer. Further studies with a larger sample size may aid in the verification of these results.

Previous studies have demonstrated that patients with cancer have an elevated REE. Bosaeus et al (19) identified that the REE was $>110 \%$ of normal levels in $48 \%$ of patients, and this tendency was dependent on the type of tumor. The REE in lung cancer patients was significantly higher than that of the control group, whereas the REE in patients with gastrointestinal cancer was not significantly increased. For patients with advanced-stage cancer, the tumor burden, psychological pressure, malaise, diminished caloric intake and loss of appetite are primary reasons for cachexia and malnutrition. In the present study, the incidence of sarcopenia among patients with advanced-stage cancer was higher than that among patients with cancer at an earlier stage, suggesting that patients with advanced cancer are more likely to have experienced skeletal muscle consumption.

Surgery, chemotherapy and radiotherapy are three traditional treatments for various types of cancer. As the preferred method of treatment, surgery can achieve resection of the tumor to avoid skeletal muscle consumption originating from the tumor burden (24). However, a significant association between surgical cancer treatment and the development of sarcopenia was not observed in the present study. Chemotherapy may also reduce the tumor burden, but the therapeutic effect is accompanied by severe cytotoxicity, causing symptoms that include vomiting and mucosal damage, which increase the body's nutritional requirements $(25,26)$. The data obtained from the current study demonstrated that the incidence of sarcopenia in patients receiving chemotherapy was slightly lower than that in patients receiving other treatments, but the difference was not significant. Such findings suggest that when regarding the prevention of sarcopenia, the effect of chemotherapy is complicated. Radiotherapy, as a localized treatment method, induces similar effects by reducing the tumor burden, but its side effects, including mucosal 
inflammation, also affect nutritional intake (26). In the present study, treatment with radiotherapy was also not significantly correlated with the development of sarcopenia. Overall, planning a safe treatment strategy for patients with cancer is an individualized, comprehensive task, with tumor progression and various interventions affecting a patient's nutritional intake and metabolism. According to the results of the current study, male gender and a reduced lymphocyte count are major risk factors for sarcopenia. However, these findings should be further verified in a clinical study with a larger sample size.

Sarcopenia may decrease the tolerance of a patient to cancer treatments. Although the results were not statistically significant, more severe complications, including infection, diarrhea and myelosuppression, were detected among cancer patients with sarcopenia in the present study, which is consistent with previous findings (16). The serum albumin level as a marker of nutritional condition is associated with several factors. A significant association between the albumin level and the development of sarcopenia was not observed, which was consistent with previous findings (16). However, the current study is the first to demonstrate that patients with sarcopenia had a significantly decreased number of lymphocytes compared with patients without sarcopenia, and that patients with cancer with an ECOG score $>2$ had a significantly increased risk of developing sarcopenia.

In conclusion, the incidence of sarcopenia among patients with cancer is high, particularly among male patients. Further studies with larger sample sizes may aid in verifying the results obtained in the present study. Nevertheless, it is necessary to prevent treatment-associated complications, including infection, diarrhea and myelosuppression, during the treatment of cancer patients with sarcopenia.

\section{References}

1. Ryall JG, Schertzer JD and Lynch GS: Cellular and molecular mechanisms underlying age-related skeletal muscle wasting and weakness. Biogerontology 9: 213-228, 2008.

2. Visser M: Towards a definition of sarcopenia - results from epidemiologic studies. J Nutr Health Aging 13: 713-716, 2009.

3. Cruz-Jentoft AJ, Baeyens JP, Bauer JM, Boirie Y, Cederholm T, Landi F, Martin FC, Michel JP, Rolland Y, Schneider SM, et al; European Working Group on Sarcopenia in Older People: Sarcopenia: European consensus on definition and diagnosis: Report of the European working group on sarcopenia in older people. Age Ageing 39: 412-423, 2010.

4. Mitchell WK, Williams J, Atherton P, Larvin M, Lund J and Narici M: Sarcopenia, dynapenia, and the impact of advancing age on human skeletal muscle size and strength; a quantitative review. Front Physiol 3: 260, 2012.

5. Tisdale MJ: Pathogenesis of cancer cachexia. J Support Oncol 1: 159-168, 2003.

6. Janssen I, Shepard DS, Katzmarzyk PT and Roubenoff R: The healthcare costs of sarcopenia in the United States. J Am Geriatr Soc 52: 80-85, 2004.

7. Wingo PA, Ries LA, Rosenberg HM, Miller DS and Edwards BK: Cancer incidence and mortality, 1973-1995: A report card for the U.S. Cancer 82: 1197-1207, 1998.
8. Brenner H: Long-term survival rates of cancer patients achieved by the end of the 20th century: A period analysis. Lancet 360 : 1131-1135, 2002.

9. Gutierrez EG, Banks WA and Kastin AJ: Murine tumor necrosis factor alpha is transported from blood to brain in the mouse. J Neuroimmunol 47: 169-176, 1993.

10. DeWys WD: Anorexia as a general effect of cancer. Cancer 43 (Suppl): 2013-2019, 1979

11. Reeds PJ, Fjeld CR and Jahoor F: Do the differences between the amino acid compositions of acute-phase and muscle proteins have a bearing on nitrogen loss in traumatic states? J Nutr 124: 906-910, 1994.

12. Deans DA, Wigmore SJ, Gilmour H, Paterson-Brown S, Ross JA and Fearon KC: Elevated tumour interleukin-lbeta is associated with systemic inflammation: A marker of reduced survival in gastro-oesophageal cancer. Br J Cancer 95: 1568-1575, 2006

13. Fearon KC: The Sir David Cuthbertson Medal Lecture 1991. The mechanisms and treatment of weight loss in cancer. Proc Nutr Soc 51: 251-265, 1992.

14. Bryant D, Becker L, Richardson J, Shelton J, Franco F, Peshock R, Thompson $\mathrm{M}$ and Giroir B: Cardiac failure in transgenic mice with myocardial expression of tumor necrosis factor-alpha. Circulation 97: 1375-1381, 1998.

15. Ramos EJ, Suzuki S, Marks D, Inui A, Asakawa A and Meguid MM: Cancer anorexia-cachexia syndrome: Cytokines and neuropeptides. Curr Opin Clin Nutr Metab Care 7: 427-434, 2004.

16. Donohoe CL, Ryan AM and Reynolds JV: Cancer cachexia: Mechanisms and clinical implications. Gastroenterol Res Pract 2011: 601434, 2011.

17. Mourtzakis M, Prado CM, Lieffers JR, Reiman T, McCargar LJ and Baracos VE: A practical and precise approach to quantification of body composition in cancer patients using computed tomography images acquired during routine care. Appl Physiol Nutr Metab 33: 997-1006, 2008.

18. Shen W, Punyanitya M, Wang Z, Gallagher D, St-Onge MP, Albu J, Heymsfield SB and Heshka S: Total body skeletal muscle and adipose tissue volumes: Estimation from a single abdominal cross-sectional image. J Appl Physiol (1985) 97: 2333-2338, 2004.

19. Bosaeus I, Daneryd P, Svanberg E and Lundholm K: Dietary intake and resting energy expenditure in relation to weight loss in unselected cancer patients. Int J Cancer 93: 380-383, 2001.

20. Antoun S, Baracos VE, Birdsell L, Escudier B and Sawyer MB: Low body mass index and sarcopenia associated with dose-limiting toxicity of sorafenib in patients with renal cell carcinoma. Ann Oncol 21: 1594-1598, 2010.

21. Prado CM, Lieffers JR, McCargar LJ, Reiman T, Sawyer MB, Martin L and Baracos VE: Prevalence and clinical implications of sarcopenic obesity in patients with solid tumours of the respiratory and gastrointestinal tracts: A population-based study. Lancet Oncol 9: 629-635, 2008.

22. Poortmans JR, Carpentier A, Pereira-Lancha LO and Lancha Jr A: Protein turnover, amino acid requirements and recommendations for athletes and active populations. Braz J Med Biol Res 45: 875-890, 2012.

23. Pahor M and Kritchevsky S: Research hypotheses on muscle wasting, aging, loss of function and disability. J Nutr Health Aging 2: 97-100, 1998.

24. Williams JP, Phillips BE, Smith K, Atherton PJ, Rankin D, Selby AL, Liptrot S, Lund J, Larvin M and Rennie MJ: Effect of tumor burden and subsequent surgical resection on skeletal muscle mass and protein turnover in colorectal cancer patients. Am J Clin Nutr 96: 1064-1070, 2012.

25. Akbulut G: New perspective for nutritional support of cancer patients: Enteral/parenteral nutrition. Exp Ther Med 2: 675-684, 2011.

26. Santarpia L, Contaldo F and Pasanisi F. Nutritional screening and early treatment of malnutrition in cancer patients. J Cachexia Sarcopenia Muscle 2: 27-35, 2011. 